\title{
The ATLAS Strip Detector System for the High Luminosity LHC
}

\author{
Jan-Hendrik Arling, on behalf of the ATLAS Collaboration
}

\begin{abstract}
The ATLAS experiment at the Large Hadron Collider (LHC) is currently preparing for major detector upgrades for the Phase-II of the LHC operation, scheduled to start in 2026. In order to achieve an integrated luminosity up to $4000 \mathrm{fb}^{-1}$ within the High Luminosity LHC (HL-LHC) phase, the instantaneous luminosity is expected to reach unprecedented values. To cope with the expected radiation damage and high density of tracks per bunch crossing, a complete replacement of the existing Inner Detector of ATLAS is required.

An all-silicon Inner Tracker (ITk) is under development with a pixel detector subsystem surrounded by the strip detector subsystem at larger radii, aiming to provide an increased tracking coverage up to pseudorapidities of four.

In this report an overview of the strip detector system design of the ITk is given as well as the results from an extensive prototyping effort are presented. The main focus is on recent testbeam results of the validation of the performance of unirradiated and irradiated strip detector modules. These are able to reach the specified performance requirements in the HL-LHC environment. The next step of the ITk project is the transition to the production phase to provide a functional detector by 2026 .
\end{abstract}

\section{INTRODUCTION}

$\mathbf{T}$ HE ATLAS detector [1] at the Large Hadron Collider [2] has successfully finished its Run- 2 data-taking by the end of 2018 , collecting in total more than $175 \mathrm{fb}^{-1}$ of proton-proton collision data at energies of upto $\sqrt{s}=13 \mathrm{TeV}$. In the time from 2024 to 2026, the LHC complex will be upgraded to the High Luminosity LHC (HL-LHC), to deliver an expected instantaneous luminosity of up to $7.5 \times 10^{34} \mathrm{~cm}^{-2} \mathrm{~s}^{-1}$, accumulating to a total integrated luminosity of up to $4000 \mathrm{fb}^{-1}$ per experiment. On the one hand, the huge statistics of recorded collision data opens up the opportunities to perform precise measurements of the Standard Model of particle physics (SM) as well as searches for physics beyond the SM. On the other hand, the HL-LHC environment poses significant challenges to the experiments, most notably the pileup will reach average values of $200 p p$ interactions per bunch crossing and the radiation damage in the detectors is expected to be a factor ten higher due to hadron fluences of up to $2 \times 10^{16} \mathrm{n}_{\mathrm{eq}} / \mathrm{cm}^{2}$.

The current tracking system of ATLAS, the Inner Detector [3], designed for the LHC conditions will not be able to cope with the challenging conditions of the HL-LHC. Therefore, the system will be replaced by the new Inner Tracker (ITk) before 2026. The ITk will be an all-silicon tracking

Manuscript received December 13, 2019.

Copyright 2019 CERN for the benefit of the ATLAS Collaboration. Reproduction of this article or parts of it is allowed as specified in the CC-BY-4.0 license.

J.-H. Arling is with the Deutsches Elektronen-Synchrotron (DESY), Notkestr. 85, 22607 Hamburg, Germany (e-mail: jan-hendrik.arling@ desy.de). detector, consisting of a pixel subsystem [4] in the region closest to the beam pipe, and a strip subsystem [5] at higher radii. The design layout of the ITk detector, depicted as a quarter segment, is shown in Fig. 1. As shown in the layout, the detector can be separated in a barrel region, located centrally around the interaction point, and the end-cap region covering the forward direction. Here, the pixel barrel will consist of five layers, with inclined sensor modules in the forward region, and the pixel end-caps are formed by individually located rings, accumulating to roughly $14 \mathrm{~m}^{2}$ of silicon. For the strip detector, four barrel layers and end-caps consisting of six disks are planned, with a total silicon area of about $165 \mathrm{~m}^{2}$. With the ITk, the tracking coverage will be increased from the current Inner Detector pseudorapidty of 2.5 to 4.0. Due to the increased granularity, a reduced material budget and more radiation-hard sensors and electronics, the ITk will maintain or improve the tracking performance of ATLAS even in the challenging HLLHC environment.

In the following, the constituents of the ITk Strip Detector will be introduced, showing thereafter results of the prototyping phase and concluding with an outlook to the production phase of the ITk.

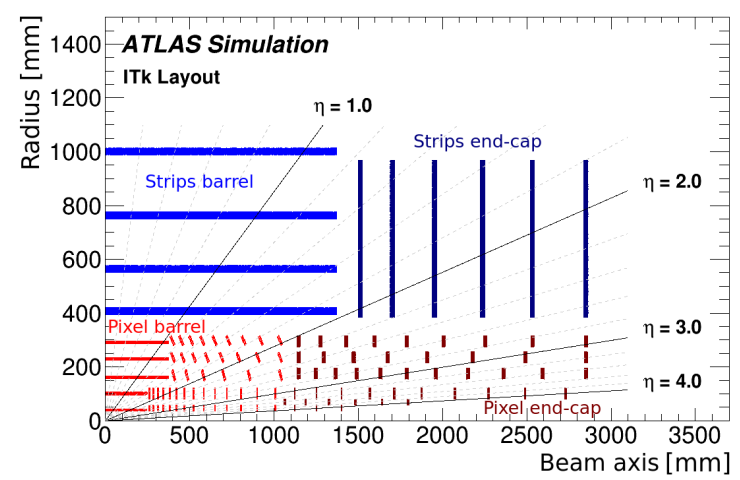

Fig. 1. Layout for the ATLAS ITk upgrade, shown as one quarter of the detector with the interaction point at zero, the beam line aligned along the horizontal axis and the radius as the vertical axis. The detector is separated in the barrel and end-cap regions, where the pixel subsystem is shown in red while the strip subsystem is shown in blue. Modified from [6].

\section{OVERVIEW OF THE ITK STRIP Detector}

The ITk Strip Detector employs a highly modular design to facilitate the multi-site construction and production plan for the different components building up the detector. For this, the silicon strip sensor together with readout and power structures build up a silicon module as the smallest assembled structure. 
These are mounted on top of lightweight support structures (called staves in the barrel and petals in the end-cap), which will be integrated in the global structures (called cyclinders in the barrel and disks in the end-cap). The full chain from sensor to the final detector subsystem is discussed in the following.

\section{A. Strip sensors}

The chosen technology for the silicon strip sensors is the $\mathrm{n}^{+}$in-p Float Zone technology [7]. This decision was driven by the expected hadron fluences of up to $1.6 \times 10^{15} \mathrm{MeVn}_{\mathrm{eq}} / \mathrm{cm}^{2}$ (including a safety factor of 1.5), as $\mathrm{n}^{+}$-in-p sensors (contrary to the currently used $\mathrm{p}$-in-n) do not suffer from the radiationinduced type inversion. Moreover, using electrons as charge carriers allow a stronger and faster signal as well as less charge trapping. The inter-strip isolation is achieved by using p-stop implants. The sensors are 300-320 $\mu \mathrm{m}$ thick and made on 6inch wafers in single-sided production. A schemativ view of the sensor is shown in Fig. 2.

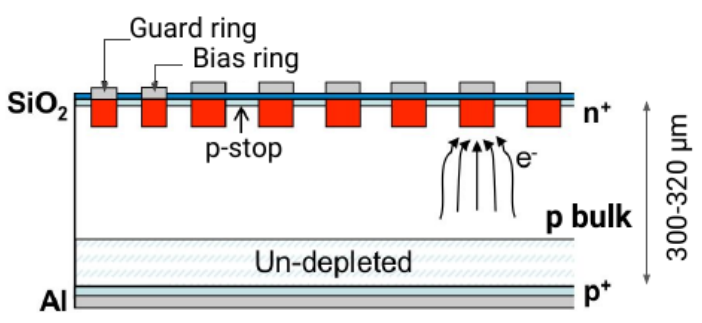

Fig. 2. Schematic cross section of the ITk strip silicon sensors using $n^{+}$-in-p technology; this allows good signal even for under-depletion.

For the barrel part, rectangular sensors with approximately $10 \times 10 \mathrm{~cm}^{2}$ are used, with either two or four rows of strips, called long-strip (LS) and short-strip (SS) respectively. The strip pitch is $75.5 \mu \mathrm{m}$ with a strip length of $24.1 \mathrm{~mm}$ for SS and $48.2 \mathrm{~mm}$ for LS. In the end-cap, six different annular sensor shapes are used in order to allow $\mathrm{r}-\phi$-coverage with radial strips pointing to the beam axis, named R0 to R5 from the inner to the outer. Due to the expected track density, the strip length is varying from 15.1 to $60.2 \mathrm{~mm}$ and the strip pitch from 60.9 to $80.7 \mu \mathrm{m}$. To enable three-dimensional spacepoint reconstruction by the double-sided population of the support structures, a stereo rotation between the strips of back-to-back sensors is introduced. In the barrel, the sensors are rotated by \pm 26 mrad during placement, whereas in the end-cap the strips themselves are skewed to point slightly off-center with a stereo angle of $\pm 20 \mathrm{mrad}$.

\section{B. ASICs and electronics}

The signal charge created by a passing charged particle through the silicon sensor is converted to a hit by the front-end chip, called ATLAS Binary Chip (ABCStar). This binary ASIC with an analogue preamplifier-shaper processes the signals from 256 connected strips. The hybrids [8], polyimide-based printed circuit boards (PCB), groups a number of ASICs together for reading out one sensor segment. The hit information from these chips is transferred to the Hybrid Controller Chip (HCCStar), which serves as an interface to the service bus and is placed also on the hybrids. The powerboard is another PCB containing a DC-DC converter [9], the radiation-hard bPOL12V, for supplying the desired low voltage of $1.5 \mathrm{~V}$ to the ASICs by reducing the input voltage of $11 \mathrm{~V}$. By the use of the DC-DC converter, ohmic losses in the cables are reduced while minimizing the material budget. The task of power control and operation monitoring is taken over by the Autonomous Monitor \& Control Chip (AMAC). In addition, the high-voltage bias filter and switch is contained.

All the ASICs are built in $130 \mathrm{~nm}$ CMOS technology, and the final versions of chips became available only in late 2018. Therefore, some of the results are obtained with prototypes equipped with the previous chipsets, called the ABC130 and HCC130, using a serial readout scheme, but are otherwise comparable to the final Star chipsets.

\section{Strip modules}

The silicon strip module is the most basic unit, which consists of the following components introduced already: a single strip sensor, one or two hybrid circuit boards, and a powerboard, with a schematic view depicted in Fig. 3.

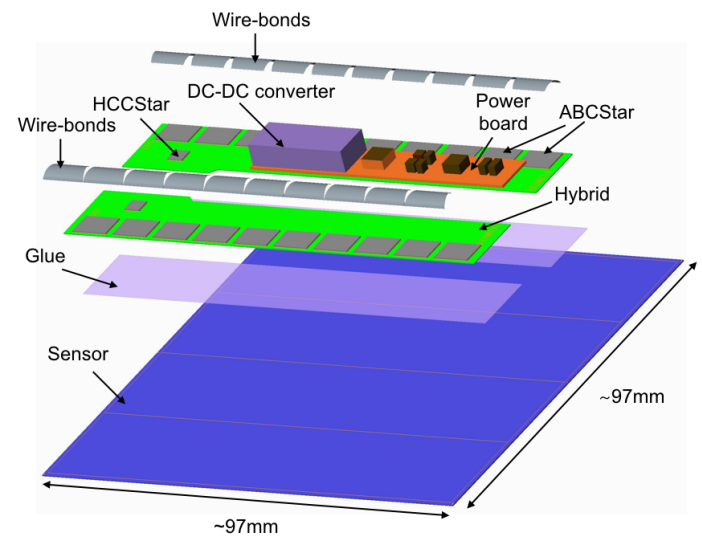

Fig. 3. Exploded schematic of a silicon strip module with the wire-bonds connecting the strips to the front-end electronics (grey). The hybrid (green) hosting the readout ASICs and the power board (orange) are directly glued on the silicon strip sensor (blue). [5]

In total for barrel and end-cap, 17,888 modules are required for the full ITk strip detector. A set of the prototype modules, consisting of the barrel modules with short- and long-strips as well as the innermost end-cap module R0, are shown in Fig. 4. Due to the high number of required modules, a mass production scheme with dedicated tools for module assembly was created, with the ability to produce and test the single units independently at different sites throughout the collaboration.

\section{Local support structures}

The modules are glued directly on both sides of local support structures, consisting of low-mass carbon-fiber (CF) sandwich structures [10]. Theses cores are made up from a $\mathrm{CF}$ facesheet, which is co-cured together with the bus-tape. The bustape is a polyimide circuit structure providing power and data lines to the modules. To provide stability to the structure, lowdensity CF honeycomb is glued on the face-sheet, where the 

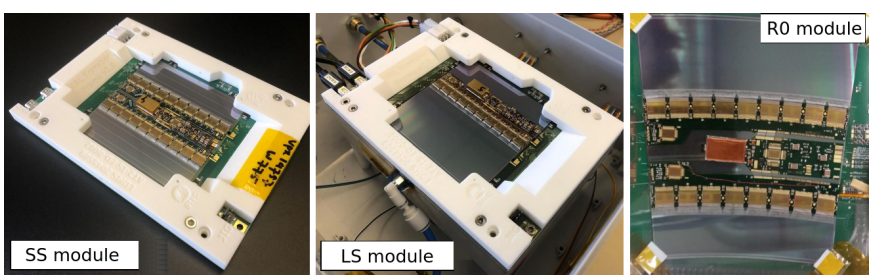

Fig. 4. Examples of ITk strip modules on dedicated testframes for module testing: short-strip barrel (left), long-strip barrel (middle) and end-cap R0 (right). Visible are the hybrids, with the ABC and HCC ASICs, and the powerboard, with the DC-DC converter below a shield box, glued on top of the silicon sensor.

sides of the core are sealed with close-outs. Cooling of the modules is ensured by dual-phase $\mathrm{CO}_{2}$ cooling at a design temperature of $-35^{\circ} \mathrm{C}$ through Titanium cooling pipes, which are surrounded by a high-conductivity thermal foam inside the sandwich structure, closed up by the backside face-sheet. The schematic cross section through a core, common for both barrel and end-cap geometries, is shown in Fig. 5.

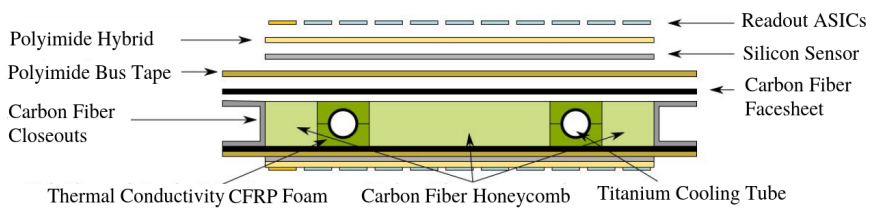

Fig. 5. Schematic cross section of a local support structure for the ITk Strip Detector. The core structure is optimized for minimizing the material while supplying high stability for the silicon modules glued on top. The embedded Titanium pipe allows for efficient dual-phase $\mathrm{CO}_{2}$ cooling and the polyimide bus-tape is the power and data bus of the structure. [5]

The outcome of this conceptual design is a thin support structure, with a short thermal path $(\sim 3 \mathrm{~mm})$ and reduced material budget $(\mathrm{x} / \mathrm{X} 0 \sim 2 \%)$. Moreover, the structure is relatively simple to allow large scale reproducibility, with the ability again to test cores individually before assembly.

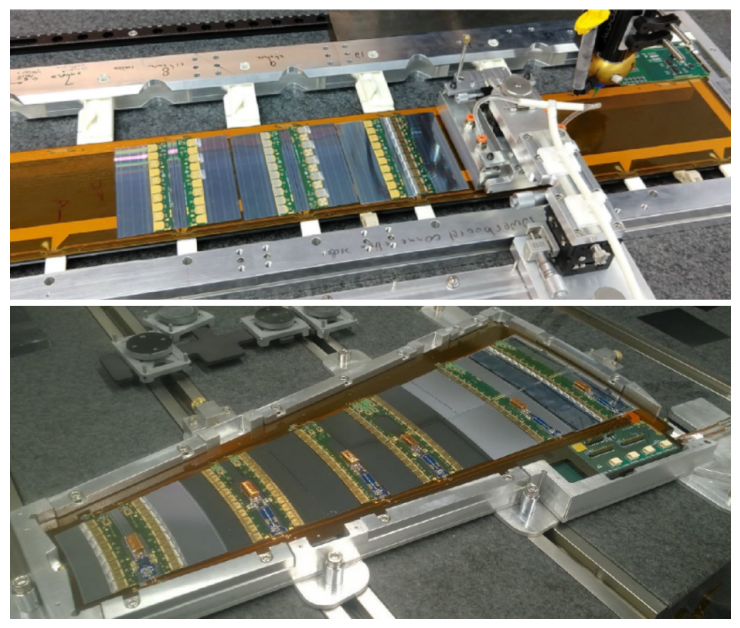

Fig. 6. Semi-electrical stave during module loading (top) and fully-loaded semi-electrical petal in loading frame (bottom).

The local supports have two designs: for the barrel the stave and for the end-cap the petal. On the one hand, a stave is a rectangular core with a length of $1.4 \mathrm{~m}$, populated by 14 rectangular modules aligned on each side. On the other side, the petal is a wedge-shaped core of $0.6 \mathrm{~m}$ length, populated with six trapezoidal-shaped modules on each side. In Fig. 6 prototypes of the stave and petal are shown, which are in the semi-electrial version, meaning that the modules are not yet real, fully functional silicon strip modules.

\section{E. Global support structures}

The staves and petals are inserted into the global support structures, called cyclinders for the barrel and disks in the end-caps. The global supports are made also primarily from carbon fiber materials to reduce the material budget as much as possible while providing support across a wide operational temperature range of the detector. All services, like cooling pipes and electrical connections, are connected at the end of the structures, to reduce the amount of material inside the tracking detector volume. The barrel subsystem is made up of four concentric cylinders connected together at the ends of the cylinders by interlinks. In the end-caps the petals are mounted onto wheel structures, forming a disk populated with petals from both sides, and one end-cap containing six disks. The global support structures are depicted in Fig. 7.

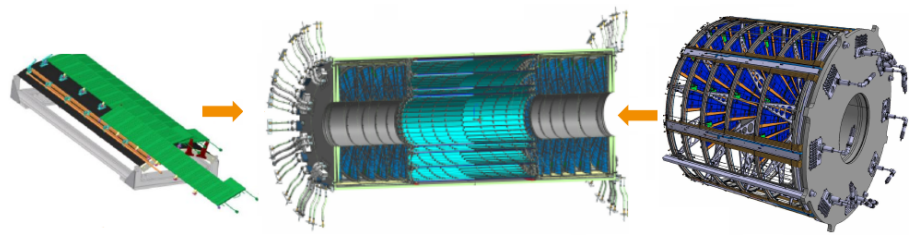

Fig. 7. Staves are inserted into the cyclinders (left) and petals inserted into the disks (right), forming the barrel and end-cap structure of the ITk strip detector, respectively. [5]

The total number of required units and structures, starting from the silicon strip modules to the local and global support structures, building up the barrel and the two end-caps of the ITk strip detector, are summarized in Table I.

\begin{tabular}{lcccc}
$\begin{array}{l}\text { Barrel } \\
\text { Layer: }\end{array}$ & $\begin{array}{c}\text { Radius } \\
{[\mathbf{m m}]}\end{array}$ & $\begin{array}{c}\text { \# of } \\
\text { staves }\end{array}$ & $\begin{array}{c}\text { \# of } \\
\text { modules }\end{array}$ & $\begin{array}{c}\text { Area } \\
{\left[\mathbf{m}^{2}\right]}\end{array}$ \\
\hline L0 & 405 & 28 & 784 & 7.49 \\
L1 & 562 & 40 & 1120 & 10.7 \\
L2 & 762 & 56 & 1568 & 14.98 \\
L3 & 1000 & 72 & 2016 & 19.26 \\
Total half barrel & & 196 & 5488 & 52.43 \\
Total barrel & & 392 & 10976 & 104.86 \\
\hline End-cap & z-pos. & \# of & \# of & Area \\
Disk: & {$[\mathbf{m m}]$} & petals & modules & {$\left[\mathbf{m}^{2}\right]$} \\
\hline D0 & 1512 & 32 & 576 & 5.03 \\
D1 & 1702 & 32 & 576 & 5.03 \\
D2 & 1952 & 32 & 576 & 5.03 \\
D3 & 2252 & 32 & 576 & 5.03 \\
D4 & 2602 & 32 & 576 & 5.03 \\
D5 & 3000 & 32 & 576 & 5.03 \\
Total one EC & & 192 & 3456 & 30.2 \\
Total ECs & & 384 & 6912 & 60.4 \\
\hline Total & & $\mathbf{7 7 6}$ & $\mathbf{1 7 8 8 8}$ & $\mathbf{1 6 5 . 2 5}$ \\
\hline
\end{tabular}

TABLE I

Number OF MODULES AND STAVES/PETALS FOR THE ITK STRIP DeTECTOR [5]. 


\section{PROTOTYPING RESULTS}

Intense prototype building and testing were conducted over the last years to finalize the designs and find the best options to fulfill the ITk detector specifications. Here, all sub-components from the silicon module to the support structures were tested and evaluated, which is possible due to the highly modular design of the detector system discussed before. In the following, some of the results for performance evaluation of the silicon strip sensors and the strip modules will be shown.

\section{A. Strip sensor testing}

One of the biggest challenges for the ITk detector are the harsh running conditions of the HL-LHC in terms of expected radiation levels. As example, Fig. 8 shows the expected fluence simulations at the detector's end-of-life.

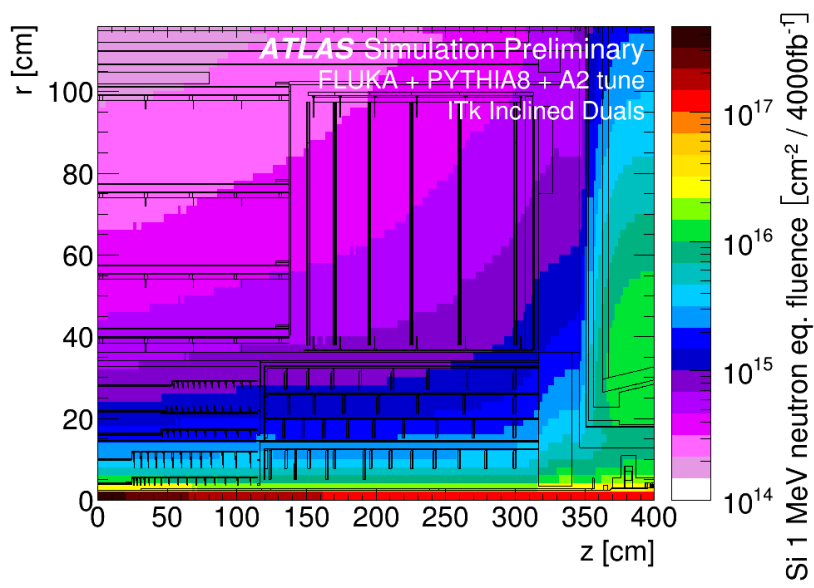

Fig. 8. Simulation of expected fluence in terms of $1 \mathrm{MeV}$ equivalent flux for the ITk detector layout, shown as one quadrant of the detector with the interaction point at zero, the beam line aligned along the horizontal axis and the radius as the vertical axis [11].

To cope with this, the silicon sensors must withstand a hadron fluence of up to $1.6 \times 10^{15} \mathrm{MeV} \mathrm{n}$ eq $/ \mathrm{cm}^{2}$ and a total ionising dose of $66 \mathrm{MRad}$, with a safety factor of 1.5 included. For sensor characterization, extensive irradiation campaigns, using protons, neutrons, pions and gammas, have been carried out to ensure the sensor performance at end-of-life. A typical measurement to check the performance of the irradiated sensors is to measure the charge collection efficiency in a beta source test setup.

In Fig. 9, the collected charge as a function of the fluence for different forms of irradiation and sensor design stages (prototypes from submissions in 2007 and 2012) at a bias voltage of $500 \mathrm{~V}$ is depicted. The drawn vertical line shows the maximum expected fluence as specified before, at which it is visible that a signal of more than 10,000 electrons is still reached. Compared to expected noise values of well below 1,000 electrons, the signal-to-noise ratio is better than ten and therefore inside the ITk specifications. The same kind of test was repeated with the newest set of prototypes and different irradiations in 2019, showing good agreement for neutron and fair agreement for proton irradiation compared to the averaged results of the 2012 sensor prototypes.

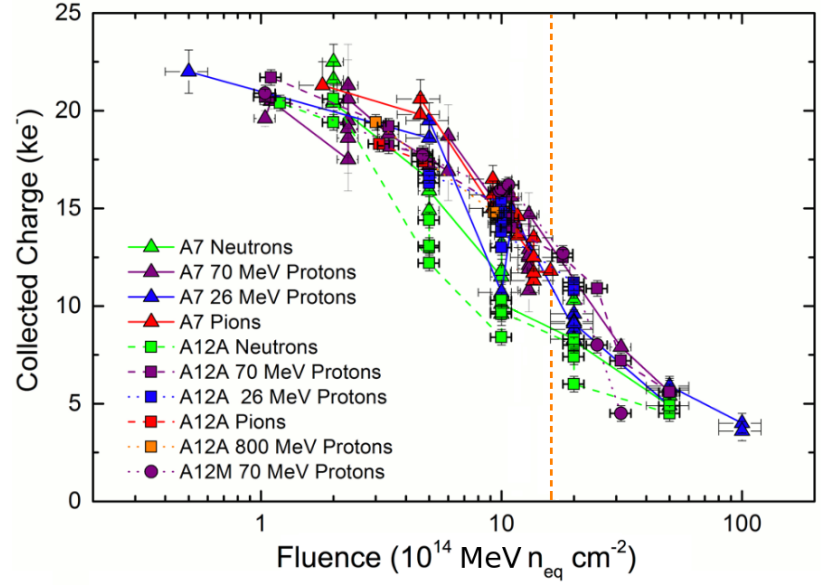

Fig. 9. Collected charge in beta source measurements for various prototype miniature sensors, after irradiation of different types and to different fluences. The sensors are biased to the design voltage of $500 \mathrm{~V}$. A7 and A12 in the legend refer to sensors delivered in 2007 and 2012, respectively, while A12M includes stereo rotated strips. The drawn vertical line indicates the maximum fluence expected at the end-of-life of the ITk detector. Modified from [5].

\section{B. Strip module testing}

The performance of prototype silicon strip modules was extensively studied in 2018 and 2019 using high-energy testbeam facilities, either with the electron beam of up to $6 \mathrm{GeV}$ energy at DESY [12] or with pions of up to $120 \mathrm{GeV}$ at CERN SPS. Tracking is provided by the EUDET-type beam telescopes [13] with six MIMOSA26 sensor planes with a resolution of 5$10 \mu \mathrm{m}$ at DESY and $3-5 \mu \mathrm{m}$ at CERN, with the reconstruction performed with the EUTelescope framework [14]. Moreover, a dedicated timing layer (either ATLAS FE-I4 pixel or Alpide sensor plane) is used for time resolution higher than the telescope integration time, to match the telescope tracks with the faster hits on the tested device (DUT). A typical testbeam setup is shown in Fig. 10.

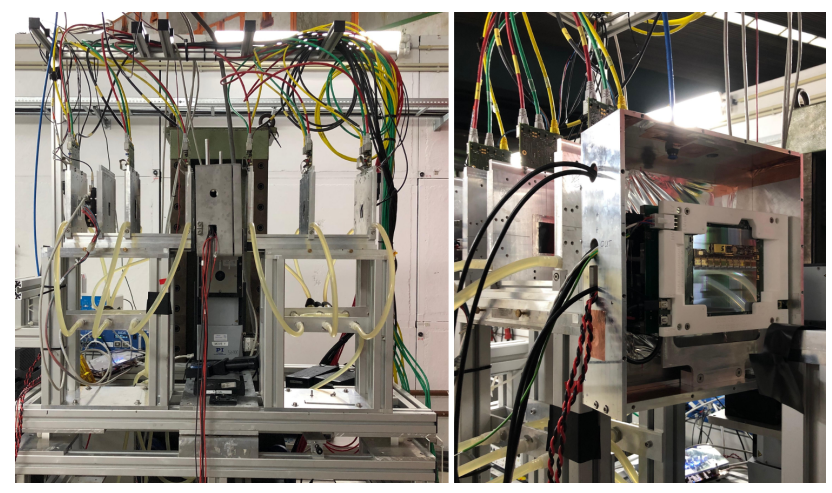

Fig. 10. Testbeam setup with the EUDET-type telescope with six MIMOSA26 sensor planes and an additional timing layer surrounding a test box with the mounted DUT inside (left). The opened box shows a barrel long-strip module supplied with power, data readout, nitrogen flushing and cooling (right).

\section{Un-irradiated long-strip module}

The first test of un-irradiated barrel long-strip module with prototype chipset ABC130/HCC130 happened at DESY with $4 \mathrm{GeV}$ electrons in June 2018. A typical result is to measure the hit efficiency of the module as function of its selected 
threshold, which shows in this test the expected behavior of an S-curve and for the un-irradiated case also a wide range of working points resulting in a required high efficiency. Only small variations in the curves for the different readout ASICs were observed, which can be accounted for by differences in the calibration between single readout ASICs. Therefore, the tested LS module showed the expected behavior in the testbeam characterization.

\section{Back-to-back RO}

Tested in the same testbeam campaign were two ITk strip endcap modules (R0) mounted back-to-back onto a small scale support core structure including services, mimicking the effect of the final petal core. Both R0 modules were equipped with the ABC130/HCC130 chipset. A picture of the assembled module on the support structure is depicted in Fig. 11.

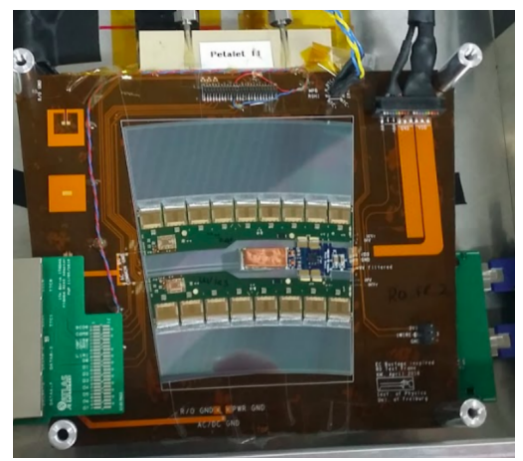

Fig. 11. Back-to-back mounted R0 end-cap modules on carbon fiber support structure including a cooling pipe, glued directly on top of bus-tape.

Here, the three-dimensional space reconstruction using the built-in stero angle could be demonstrated. For this, Fig. 12 shows the radial residual of the measured telescope track position and the hit in the DUT along the strip, with a width of $\sigma_{r}=1.1 \mathrm{~mm}$. This result is reasonable, taking into account that only two sensor planes are used and the fact that the assembly of the structure was done by hand, resulting in the non-perfect design stereo angle of $40 \mathrm{mrad}$.

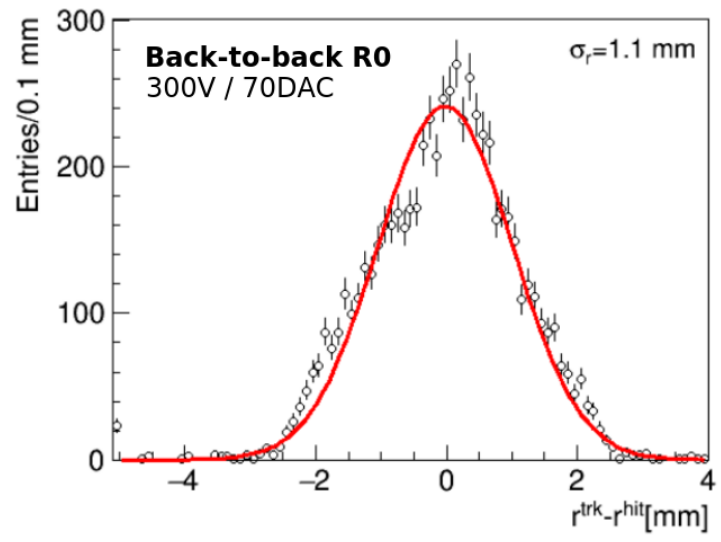

Fig. 12. Radial residual along the strip direction of back-to-back R0 modules showing the possbility of three-dimensional space reconstruction with the stereo angle. The width of the residual distribution is calculated as width of a Gaussian fit. [15]

\section{Irradiated ROStar}

The evaluation of the expected end-of-lifetime performance of the modules after irradiation is a critical input for the design verification. The ITk specifications for the range of operating thresholds of the module after the end-of-life fluence are a hit detection efficiency above $99 \%$ and a noise occupancy below $0.1 \%$ at the same time. In 2016 for the Technical Design Report, the tested prototypes showed only a very tiny operating window for the $\mathrm{ABC} 130$ chipset, which is shown in Fig. 13. Here, taking into account effects like e.g. less noisy final readout chipset, would open up the range of operational thresholds.

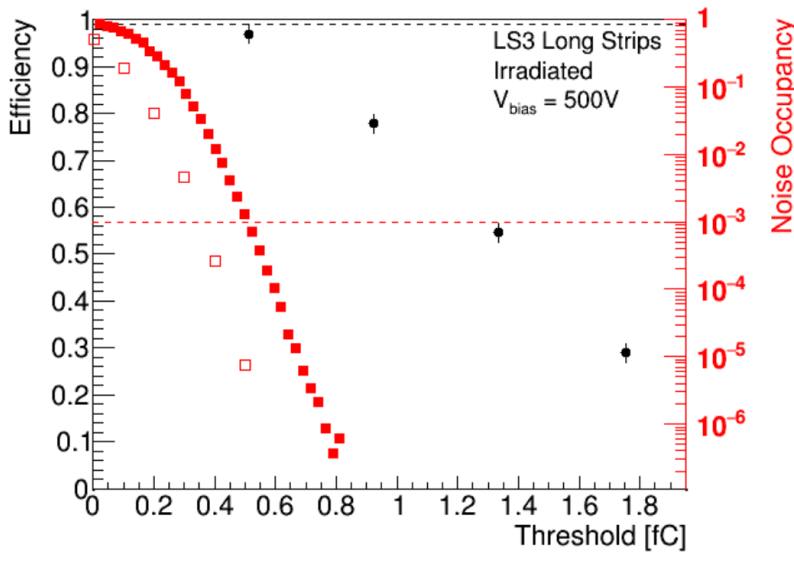

Fig. 13. The results show the irradiated long-strip module tested in 2016 operated at a bias voltage of $500 \mathrm{~V}$. The signal efficiency (black dots) and the noise occupancy at the same time at different thresholds for the ABC130 front-end (red squares) is measured. The predicted noise occupancy for the Star chipset is also shown (open squares). Possible operating windows in terms of thresholds for fulfilling the ITk requirements can be identified by the required hit efficiency as black dashed line and at the same time the criterion on the noise occupancy as red dashed line. [5]

To verify this prediction, an end-cap R0 ATLAS12 sensor was irradiated with $24 \mathrm{GeV}$ protons at CERN SPS to a NIEL fluence of $1.5 \times 10^{15} \mathrm{MeV} \mathrm{n}_{\mathrm{eq}} / \mathrm{cm}^{2}$ (the maximum expected $\mathrm{EC}$ module fluence including a safety factor of 1.4); also the hybrid with the production front-end chips, ABCStar and HCCStar, and the powerboard were irradiated at RAL with X-rays to a total ionising dose up to $35 \mathrm{MRad}$. This dose is smaller than the expected, but due to the beginning testbeam time, no longer irradiation was possible. In parallel, the noise was measured to be constant with the accumulated dose, so no major effect is expected here.

In a testbeam campaign at DESY in June 2019 the module performance after the irradiation was tested. The measured signal-to-noise ratio of 11.7 can be compared to an expected of 12.2. Moreover, thresholds around $0.5 \mathrm{fC}$ satisfy both ITk requirements in hit efficiency and noise occupancy. This, in contrast to the results of the previous prototype testing shown before, demonstrates a stable operational window of thresholds. As for the R0 module, the lowest signal-to-noise ratio is expected, the test showed the worst-case scenario, so the requirements on the module performance at the end-of-lifetime are fulfilled. 


\section{OUTLOOK TO ITK PRODUCTION}

The decisions for the technical design of the ITk Strip Detector are complete and the prototype testing shows excellent performance, so the project is in a phase transition: from the successfull R\&D phase into the production phase. As already shown in Table I, in total 392 staves and 384 petals must be constructed and populated with 17,888 modules. This effort is shared across many institutes in the ITk collaboration, with many sites constructing different parts of the detector as depicted in Fig. 14.

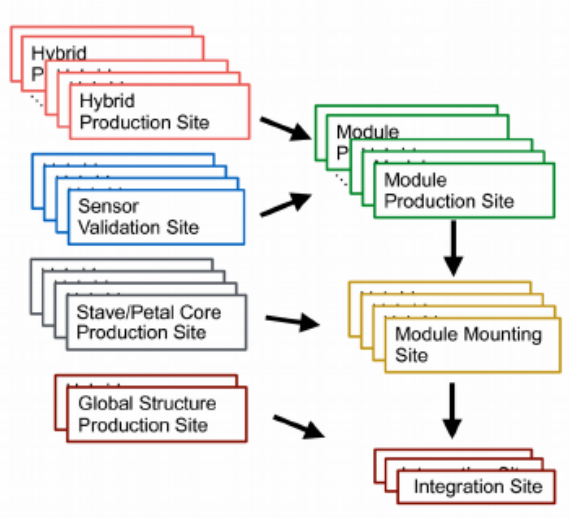

Fig. 14. The production of ITk Strip Detector parts is shared by many construction sites and due to the high modularity also by component type, such as sensors, modules and local support [5].

The first step will be to produce $5 \%$ of the required components in the pre-production phase starting now. This is used to establish procedures of assembly and testing and qualify the different construction sites. Due to its modular design, each component can be tested in advance, therefore a high focus is placed on the quality control and quality assurance procedures, ensuring a highly-functioning detector. After successfull demonstration in pre-production, the production phase will last until 2024. To be ready for recording first collision data in 2026, the detector parts will be assembled, installed and commissioned at CERN before this.

\section{CONCLUSION}

For the harsh environment of the high-luminosity phase of the LHC, the ATLAS ITk Strip Detector will provide high tracking performance, even after the expected significant radiation damage. A long $R \& D$ program has resulted in a detector design coping with these challenging conditions, as it was demonstrated in many prototype studies. In this paper, the focus was on results of silicon sensor testing and evaluating the strip module performance in testbeams. The next step is entering into the (pre-)production phase with delivering a fully functional tracking detector for the planned start of collisions in 2026. This is only possible due to the engagement of involved people in the collaboration of institutes worldwide.

\section{ACKNOWLEDGMENT}

Some of the measurements leading to these results have been performed at the Test Beam Facility at DESY Hamburg
(Germany), a member of the Helmholtz Association (HGF); and at the CERN SPS Test Beam Facility. We gratefully thank the operators of these facilities. This project has received funding from the European Union's Horizon 2020 Research and Innovation programme under Grant Agreement no. 654168.

\section{REFERENCES}

[1] ATLAS collaboration, The ATLAS Experiment at the CERN Large Hadron Collider, JINST 3 (2008) S08003. https://doi.org/10.1088/1748-0221/3/08/ s08003.

[2] L. Evans and P. Bryant, LHC Machine, JINST 3 (2008) S08001. https: //doi.org/10.1088/1748-0221/3/08/s08001.

[3] ATLAS collaboration, ATLAS inner detector: Technical Design Report, vol. $1 \& 2$, CERN-LHCC-97-016 \& CERN-LHCC-97-017 (1997). https: //cds.cern.ch/record/331063 \& https://cds.cern.ch/record/331064.

[4] ATLAS collaboration, Technical Design Report for the ATLAS Inner Tracker Pixel Detector, CERN-LHCC-2017-021 (2017). https://cds.cern. $\mathrm{ch} / \mathrm{record} / 2285585$.

[5] ATLAS collaboration, Technical Design Report for the ATLAS Inner Tracker Strip Detector, CERN-LHCC-2017-005 (2017). https://cds.cern. $\mathrm{ch} / \mathrm{record} / 2257755$.

[6] ATLAS collaboration, Expected Tracking Performance of the ATLAS Inner Tracker at the HL-LHC, ATL-PHYS-PUB-2019-014, (2019). https://cds. cern.ch/record/2669540.

[7] Y. Unno, et al., Development of n+-in-p large-area silicon microstrip sensors for very high radiation environments: ATLAS12 design and initial results, NIM A 765 (2014) 80-90. https://doi.org/10.1016/j.nima.2014.06. 086.

[8] K. Mahboubi, et al., The front-end hybrid for the ATLAS HL-LHC silicon strip tracker, JINST 9 (02) (2014) 2027. https://doi.org/10.1088/ $1748-0221 / 9 / 02 / \mathrm{c} 02027$.

[9] A. Affolder, et al., DC-DC converters with reduced mass for trackers at the HL-LHC, JINST 6 (11) (2011) 11035. https://doi.org/10.1088/1748-0221/ 6/11/c11035.

[10] S. Diez, Silicon strip staves and petals for the ATLAS Upgrade tracker of the HL-LHC, NIM A 699 (2013) 93-96. https://doi.org/10.1016/j.nima. 2012.06.004.

[11] ATLAS collaboration, Radiation Simulation Public Results: Phase II ITk Inclined Duals, April 2018, https://twiki.cern.ch/twiki/bin/view/ AtlasPublic/RadiationSimulationPublicResults.

[12] R. Diener, et al., The DESY II test beam facility, NIM A 922 (2019) 265-286. https://doi.org/10.1016/j.nima.2018.11.133.

[13] H. Jansen, et al., Performance of the EUDET-type beam telescopes, EPJTI 3 (7) (2016). https://doi.org/10.1140/epjti/s40485-016-0033-2.

[14] EUTelescope, A Generic Pixel Telescope Data Analysis Framework, https://github.com/eutelescope/eutelescope.

[15] J. Keller, The ATLAS ITk Strip Detector System for the High Luminosity LHC Upgrade, NIM A (2019) (in press). https://doi.org/10.1016/j.nima. 2019.04.007. 